\title{
A Fast Algorithm for High Quality Vector Quantization Codebook Design
}

\author{
Carlo Braccini, Fabio Cocurullo and Fabio Lavagetto \\ University of Genova, Via Opera Pia 13, I-16145 Italy
}

Tel. 39-10-3532983, Fax 39-10-3532948, e-mail Fabio.Cocurullo@dist.unige.it

\begin{abstract}
In this paper we present a new theoretical approach to the problem of optimal Vector Quantization. We base, in fact, our method on the a priori explicit analysis of the effects on the MSE distortion introduced by an arbitrary exchange of training vectors among clusters. Even when the theoretical results corresponding to the simplest possible case are used, the proposed algorithm outperforms the GLA method to an impressive extent both in speed and in performance. Experiments on different images from the USC database have proved that the proposed algorithm is 5 to 10 times faster than the GLA method increasing the convergence Peak Signal to Noise Ratio (PSNR) of up to $1.12 \mathrm{~dB}$.
\end{abstract}

\section{Introduction}

The term Vector Quantization (VQ) [1] generally indicates a class of widely used techniques for signal compression and coding within applications aiming at storage saving or transmission rate reduction. Since Lloyd and Max [2] optimal scalar quantizer, quite a lot of new proposals have been made, providing innovative algorithms for the design of vector quantizers. Their application initially, limited only to scalar signals and typically to speech, has been progressively extended to multidimensional sources like images and image sequences.

The multidimensional extension of Lloyd's approach, generally known as Lloyd-Generalized-Algorithm (GLA), iteratively modifies an initial quantizer on the basis of a predefined vector pdf or with reference to a vector training sequence: the reconstruction levels migrate to stable configurations which locally minimize a distortion functional usually based on the Mean-Square-Error (MSE). The GLA approach represents a key reference point for any new proposal concerning the design of a vector quantizer and an useful yardstick to compare its performances. The quantizer average distortion, which is locally minimized in correspondence to the GLA convergence configuration, strongly depends on the initial choice of the reconstrution levels and on the reliability of the estimated pdf [3]. Other methods have been recently proposed, including the Pairwise Nearest Neighbor (PNN) [5] and Maximum Descent (MD) algorithm [6]; the mathematical formalism of both of them can be viewed as a particular case of the more general theory presented in this paper.

In this paper we approach the problem of designing the codebook from a different point of view (substantially improving what proposed in [7] and[8]): 
after having evaluated a priori the effects produced by any possible single vector redistribution among clusters, we choose and apply the least distortion one. This way of looking at the problem with "new eyes", though quite immediate and simple in its formulation, provides powerful tools for devising a variety of new algorithms and procedures. The proposed algorithm, despite its simplicity and uniformity (there is no speed-up, no combination of different techniques), goes one step forward to solve the classical problems of convergence speed and codebook optimality.

\section{Mathematical Formalism}

The problem can be formalized as follows: given a training set TS of $n k$ dimensional input vectors

$$
T S=\left\{\boldsymbol{x}_{1}, \boldsymbol{x}_{2} \ldots, \boldsymbol{x}_{n}\right\}
$$

considered representative of the signal statistics, the task of designing the optimal Quantizer $Q^{*}$ consists of finding a set $P=\{p 1, p 2, \ldots, p h\}$ of clusters each containing $n_{p 1}, n_{p 2}, \ldots, n_{p h}$ vectors:

$$
p i=\left\{\boldsymbol{x}_{p i_{1}}, \boldsymbol{x}_{p i_{2}} \ldots, \boldsymbol{x}_{p i_{n_{p i}}}\right\} \quad i=1,2, \ldots, h
$$

in a way to minimize a distortion functional. In the following, the Square Error with respect to the training set $T S$ will be considered:

$$
D_{T S, P}=\sum_{i=1}^{n}\left\|x_{i}-Q\left(\boldsymbol{x}_{i}\right)\right\|^{2}
$$

where $Q\left(\boldsymbol{x}_{i}\right)$ represents the codeword associated to the cluster which includes $\boldsymbol{x}_{i}$. These codewords are (according to [9]) the corresponding centroids $\boldsymbol{m}_{p 1}, \boldsymbol{m}_{p 2}, \ldots, \boldsymbol{m}_{p h}$ :

$$
\boldsymbol{m}_{p i}=\frac{1}{n_{p i}} \boldsymbol{s}_{p i} \quad \text { where } \quad \boldsymbol{s}_{p i}=\sum_{j=1}^{n_{p i}} \boldsymbol{x}_{p i_{j}} \quad i=1,2, \ldots, h
$$

the global quantization distortion $D_{T S}$ can be obtained as the sum of the partial distortions introduced by each cluster:

$$
D_{T S, P}=d_{p 1}+d_{p 2}+\ldots d_{p h} \quad \text { where } \quad d_{p i}=\sum_{j=1}^{n_{p i}}\left\|\boldsymbol{x}_{p i_{j}}-\boldsymbol{m}_{p i}\right\|^{2}
$$

The generic partial distortion $d_{p i}$, being $\boldsymbol{m}_{p i}$ the centroid of the corresponding cluster $p i$ can be rewritten as:

$$
d_{p i}=\sum_{j=1}^{n_{p i}}\left\|x_{p_{j}}\right\|^{2}-\frac{\left\|s_{p i}\right\|^{2}}{n_{p i}} \quad i=1,2, \ldots, h
$$


The global quantization distortion $D_{T S}$ can be expressed as:

$$
D_{T S, P}=\sum_{i=1}^{n}\left\|x_{i}\right\|^{2}-\sum_{j=1}^{h} \frac{\left\|s_{p j}\right\|^{2}}{n_{p j}}
$$

Being $\sum_{i=1}^{n}\left\|\boldsymbol{x}_{i}\right\|^{2}$ independent from the specific clusters configuration, the problem of finding the optimal partition $P$ is that of maximizing

$$
S Q_{T S, P}=\sum_{j=1}^{h} \frac{\left\|\boldsymbol{s}_{p j}\right\|^{2}}{n_{p j}}
$$

Any different configuration of the quantizer can be reached by means of an arbitrary exchange of vectors among the clusters that can be formalized as follows: the generic cluster $p i$ will loose a subset $t i^{-}$of vectors and will receive a subset $t i^{+}$of vectors. The new clusters $\hat{p} 1, \hat{p} 2, \ldots, \hat{p} h$ obtained after the vector redistribution will be $\hat{p} i=\left(p i \backslash t i^{-}\right) \cup t i^{+}$Defining the sums and the corresponding centroids of the vectors within each subset $t 1^{-}, t 2^{-}, \ldots, t h^{-}$and $t 1^{+}, t 2^{+}, \ldots, t h^{+}$as $\boldsymbol{s}_{t 1}^{-}, s_{t 2}^{-}, \ldots, s_{t h}^{-} \quad, \quad s_{t 1}^{+}, s_{t 2}^{+}, \ldots, s_{t h}^{+}$ $, \quad \boldsymbol{m}_{t 1}^{-}, \boldsymbol{m}_{t 2}^{-}, \ldots, \boldsymbol{m}_{t h}^{-}$and $\boldsymbol{m}_{t 1}^{+}, \boldsymbol{m}_{t 2}^{+}, \ldots, \boldsymbol{m}_{t h}^{+}$, the new optimal output point for each cluster $\hat{p} 1, \hat{p} 2, \ldots, \hat{p} h$ will be:

$$
\hat{\boldsymbol{m}}_{p i}=\frac{\hat{\boldsymbol{s}}_{p i}}{\hat{n}_{p i}} \quad i=1,2, \ldots, h
$$

with

$$
\hat{n}_{p i}=n_{p i}-n_{T i}^{-}+n_{T i}^{+} \hat{s}_{p i}=s_{p i}-s_{T i}^{-}+s_{T i}^{+} i=1,2, \ldots, h
$$

The global distortion $\hat{D}_{T S, \hat{P}}$ after the cluster rearrangement will be:

$$
\hat{D}_{T S, \hat{P}}=\sum_{j=1}^{n}\left\|\boldsymbol{x}_{j}\right\|^{2}-\sum_{j=1}^{h} \frac{\left\|\hat{s}_{p j}\right\|^{2}}{\hat{n}_{p j}}
$$

In this way the distortion variation will be:

$$
\hat{D}_{T S, \hat{P}}-D_{T S, P}=\sum_{j=1}^{n}\left\|\boldsymbol{x}_{j}\right\|^{2}-\sum_{j=1}^{h} \frac{\left\|\hat{\boldsymbol{s}}_{p j}\right\|^{2}}{\hat{n}_{p j}}-\sum_{j=1}^{n}\left\|\boldsymbol{x}_{j}\right\|^{2}+\sum_{j=1}^{h} \frac{\left\|\boldsymbol{s}_{p j}\right\|^{2}}{n_{p j}}
$$

The expression of the distortion variation resulting from the cluster reorganization is found to be:

$$
\hat{D}_{T S, \hat{P}}-D_{T S, P}=\sum_{j=1}^{h}\left(\frac{\left\|\boldsymbol{s}_{p j}\right\|^{2}}{n_{p j}}-\frac{\left\|\hat{\boldsymbol{s}}_{p j}\right\|^{2}}{\hat{n}_{p j}}\right)
$$

that is to say:

$$
\hat{D}_{T S, \hat{P}}-D_{T S, P}=\sum_{j=1}^{h}\left(\frac{\left\|\boldsymbol{s}_{p j}\right\|^{2}}{n_{p j}}-\frac{\left\|s_{p j}-\boldsymbol{s}_{t j}^{-}+\boldsymbol{s}_{t j}^{+}\right\|^{2}}{\left(n_{p j}-n_{t j}^{-}+n_{t j}^{+}\right)}\right)
$$




\section{A Simple Algorithmic Implementation}

Finding the optimal vector redistribution among the clusters, i.e. the subsets $t 1^{-}, t 2^{-}, \ldots, t h^{-}$and $t 1^{+}, t 2^{+}, \ldots, t h^{+}$yielding the best value of $\hat{D}_{T S, \hat{P}}-D_{T S, P}$, is not a trivial task. Because of this complexity, the experiments reported in the following have been obtained applying (2) in the simplest case for evaluating the distortion variation obtainable when a single vector is transferred at each time. Given a vector $\boldsymbol{x}$ belonging to cluster $p i$, reclassifying it into any other cluster $p j$ provides, according to (2), the following distortion variation:

$$
\Delta_{i, j}=\frac{\left\|s_{p i}\right\|^{2}}{n_{p i}}-\frac{\left\|s_{p i}-x\right\|^{2}}{\left(n_{p i}-1\right)}+\frac{\left\|s_{p j}\right\|^{2}}{n_{p j}}-\frac{\left\|s_{p j}+x\right\|^{2}}{\left(n_{p j}+1\right)}
$$

The GLA algorithm, at each iteration, reassigns each vector $\boldsymbol{x}$ of the training set $T S$ to the cluster $P^{*}$ whose centroid $m^{*}$ has minimum distance from $\boldsymbol{x}$. On the contrary, in the proposed approach (which will be referenced in the following as $K-C L) \Delta_{i, j}$ (the distortion variation obtainable by transferring each vector $\boldsymbol{x}$ from its originary cluster $p_{i}$ to any other cluster $p j$ ) is evaluated; if none of the distortion variations $\Delta_{i, j}, j=1,2, \ldots, h, j \neq i$ is negative, $\boldsymbol{x}$ is not moved; otherwise it is transferred to $p_{j^{*}}$ corresponding to the maximum (negative) distortion variation $\Delta_{i, j^{*}}$. In this latter case the partitions $p i$ and $p j^{*}$ are immediately updated:

$$
\hat{\boldsymbol{s}}_{p i}=\boldsymbol{s}_{p i}-\boldsymbol{x}, \hat{n}_{p i}=\left(n_{p i}-1\right), \hat{\boldsymbol{s}}_{p j^{*}}=\boldsymbol{s}_{p j^{*}}+\boldsymbol{x}, \hat{n}_{p j^{*}}=\left(n_{p j^{*}}+1\right)
$$

Since any iterative algorithm needs some form of initialization, different approaches have been tried reporting some preliminary results [8] with specific reference to "random guess" technique. Better quality and repeatable codebooks may be obtained using the well-known splitting technique [1] allowing larger codebooks to be produced from smaller ones:

- the optimal partition of the Training Set $T S$ with size $2^{k=0}=1$ is obviously the single cluster $T S$;

- given a codebook of size $2^{k}$, an initial codebook of size $2^{k+1}$ may be obtained by "splitting" each cluster;

- the so obtained initial configuration is improved using an iterative algorithm.

Using the GLA algorithm, each cluster is splitted considering, together with the original centroid, $\boldsymbol{m}$, another codeword $\boldsymbol{m}+\boldsymbol{\epsilon}$ where $\boldsymbol{\epsilon}$ is a vector with small euclidean norm. In the proposed $K-C L$ method, each cluster is splitted by comparing a random selected component of each vector $\boldsymbol{x}$ to the corresponding component of the centroid $\boldsymbol{m}$. A well conceived implementation of the described algorithm provides almost the same complexity of the GLA algorithm for each iteration, since the term $-\frac{\left\|\boldsymbol{s}_{p_{i}}-\boldsymbol{x}\right\|^{2}}{\left(n_{p_{i}}-1\right)}$ from (3) can be computed only once for each vector $\boldsymbol{x}$ and the terms $\frac{\left\|\boldsymbol{s}_{p i}\right\|^{2}}{n_{p i}}$ and $\frac{\left\|\boldsymbol{s}_{p j}\right\|^{2}}{n_{p j}}$ can be memorized for each cluster. In this way only a slight increment in storage requirements is needed. 


\section{Experimental Results}

The proposed method has been tested in the context of image coding where it has shown significant performance improvements combined with faster convergence in comparison to the standard GLA algorithm. The presented results have been obtained on three $512 \times 512$ pixels monochromatic ( $8 \mathrm{bit} / \mathrm{pels}$ ) images from the USC test set: "lena", "girl" and "f16". The images have been subdivided in $4 \mathrm{x} 4$ blocks to form a training set of 16384 vectors clustered around 1024 codewords. A performance comparison between the GLA method and the K-CL algorithm is presented, in terms of MSE, In Table 1. These results have been obtained employing the splitting technique for both methods and keeping fixed the same number of iterations after each splitting operation. Far more impressive than the

\begin{tabular}{|l||r|r|r|r||r|r|r|r||r|r|r|r|}
\hline MSE & \multicolumn{1}{|c|}{ lena: It. \# each split } & \multicolumn{3}{|c||}{ f16: It. \# each split } & \multicolumn{3}{|c|}{ girl: It. \# each split } \\
\hline method & 1 & 4 & 10 & 40 & 1 & 4 & 10 & 40 & 1 & 4 & 10 & 40 \\
\hline GLA & 39.0 & 24.0 & 22.6 & 22.3 & 48.7 & 29.1 & 27.1 & 25.8 & 32.7 & 21.0 & 19.8 & 19.3 \\
K-CL & 22.3 & 19.8 & 19.5 & 19.4 & 25.9 & 22.5 & 22.3 & 22.2 & 19.5 & 17.6 & 17.3 & 17.2 \\
\hline
\end{tabular}

Table 1. Comparison between the GLA and the K-CL performances in terms of MSE on "lena", "f16" and "girl" image ( $512 \times 512$ pixel, 8 bpp, $4 \times 4$ blocks, 1024 codevectors)

performance gain in terms of MSE is the convergence speed as it results from Fig. 1, that displays the MSE curves obtained by applying the two methods until similar quality is reached. The quality of the K-CL coded image is noticeable

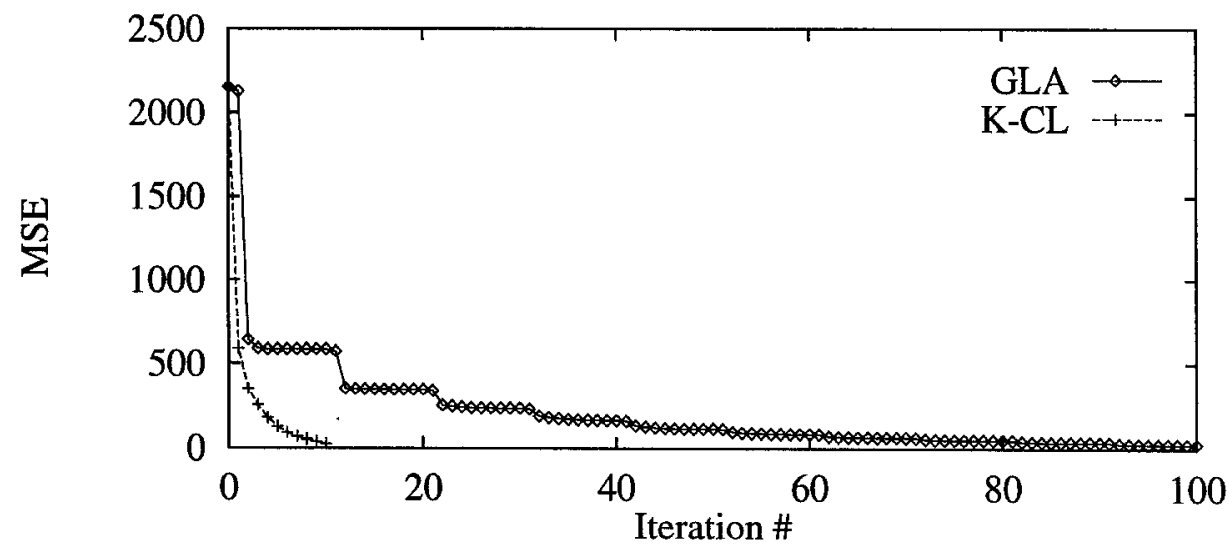

Fig. 1. Comparison of the MSE curves, versus the number of iterations, between GLA method (10 iterations after each splitting - convergence MSE=27.1) and the K-CL (1 iteration afer each splitting - convergence MSE=25.9). Both curves refer to the image "f16" which has been used as training set $(512 \times 512$ pixel, 8 bpp, $4 \times 4$ blocks, 1024 codevectors). 
higher in comparison with the GLA coded image expecially in correspondence of the high detail area. In Fig. 2 details of both coded f16 images are presented.

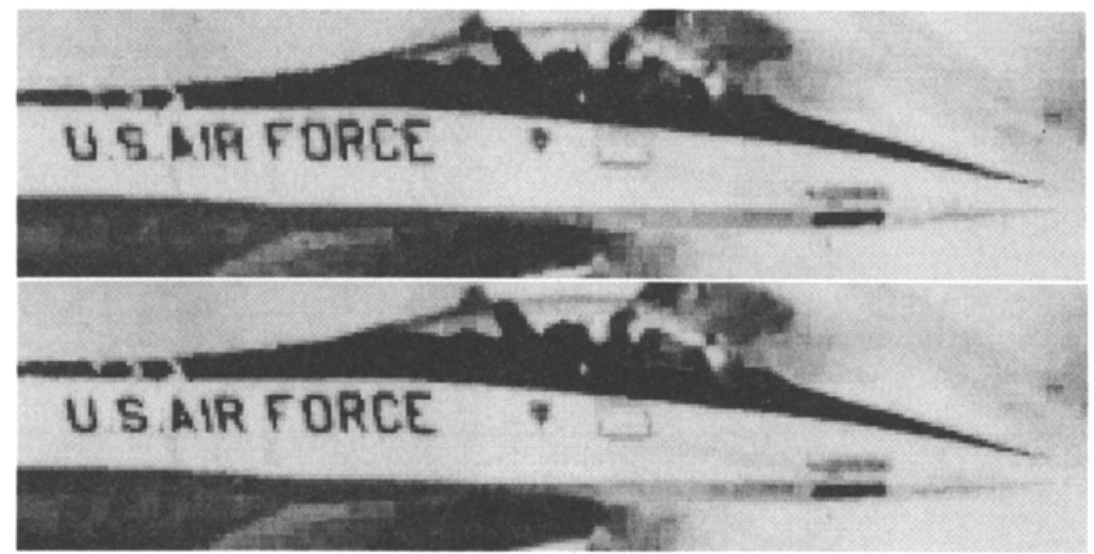

Fig. 2. Details extracted from "f16" coded images ( $512 \times 512$ pixel, 8 bpp, $4 \times 4$ blocks, 1024 codevectors 4 iteration after each splitting for both methods) via GLA (top) and $\mathrm{K}-\mathrm{CL}$ (bottom) techniques

\section{References}

1. A. Gersho, R.M. Gray, Vector Quantization and Signal Compression, Kluwer Academic Publishers, 1992.

2. J. Max, Quantizing for Minimum Distortion, IRE Trans. on Inf. Theory, Vol. IT-6, 1960, pp. 7-12.

3. R.M. Gray, E. Karnin, Multiple Local Optima in Vector Quantizers, IEEE Trans. on Inf. Theory, Vol. IT-28, 1982, pp. 256-261.

4. J.H. Conway, N.J.A. Sloane, Voronoi Regions of Lattices 2-nd Moments of Polytopes and Quantization, IEEE Trans. on Inf. Theory, Vol. IT-28, 1982, pp. 211-226.

5. W.H. Equitz, A New Vector Quantization Clustering Algorithm, IEEE Trans. Acoust. Speech Signal Processing, vol. 37, no. 10, Oct. 1989.

6. Chok-Ki Chan and Chi-Kit Ma, A Fast Method of Designing Better Codebooks for Image Vector Quantization, IEEE Trans. on Communications, Vol. 42, No. 2/3/4, Feb./Mar./Apr. 1994.

7. F. Cocurullo, F. Lavagetto and M. Moresco, Optimal Clustering for Vector Quantizer Design, Proc. EUSIPCO-92 Brusseles, Begium, August 24-27, Vol. I, pp. 563-566.

8. F. Cocurullo and F. Lavagetto, A New Algorithm for Vector Quantization, to appear in Proc. Data Compression Conference - DCC '95 Snowbird, Utah, March $28-30,1995$.

9. A. Gersho, On the Structure of Vector Quantizers, IEEE Trans. on Inf. Theory, Vol. IT-28, 1982, pp. 157,166 . 